VOL. 59 (1999) [403-408]

\title{
IMAGES OF HANKEL OPERATORS ON THE POLYDISK
}

\author{
Boo Rim Choe and Young Joo Lee
}

\begin{abstract}
We study images of Hankel operators on the Bergman or Hardy space of the polydisk. We give characterisations of pairs of antiholomorphic symbols inducing Hankel operators whose images are mutually orthogonal.
\end{abstract}

\section{RESULTS}

Let $D$ be the unit disk in the complex plane C. The unit polydisk $D^{n}$ is the cartesian product of $n$ copies of $D$. The Bergman space $A^{2}$ is the closed subspace of $L^{2}\left(D^{n}\right)=L^{2}\left(D^{n}, V\right)$ consisting of all holomorphic functions on $D^{n}$. Here, the measure $V$ is the normalised volume measure on $D^{n}$. Let $P$ be the orthogonal projection from $L^{2}\left(D^{n}\right)$ onto $A^{2}$. For a function $f \in L^{\infty}\left(D^{n}\right)$, the Hankel operator $H_{f}$ with symbol $f$ is defined by

$$
H_{f} u=u f-P(u f)
$$

for $u \in A^{2}$. Then, it is clear that $H_{f}: A^{2} \rightarrow L^{2}\left(D^{n}\right)$ is a bounded linear operator and $H_{f}\left(A^{2}\right)$ is contained in $A^{2 \perp}$, the orthogonal complement of $A^{2}$ in $L^{2}\left(D^{n}\right)$. Here,

$$
H_{f}\left(A^{2}\right)=\left\{H_{f} u: u \in A^{2}\right\}
$$

denotes the image of $H_{f}$.

On the setting of the unit disk, Wang and $\mathrm{Wu}[3]$ have recently studied the images of Hankel operators with certain antiholomorphic symbols. As a consequence of their result, we have: for a pair of bounded holomorphic functions $f$ and $g$ on $D$, the images $H_{\bar{f}}\left(A^{2}\right)$ and $H_{\bar{g}}\left(A^{2}\right)$ are mutually orthogonal if and only if either $f$ or $g$ is constant.

In this note, we first show that there are lots of pairs of nonconstant antiholomorphic symbols on the polydisk inducing Hankel operators whose images are mutually orthogonal. This follows from the following characterisation which can be viewed as an extension of the result of Wang and Wu. In what follows, we let $H^{\infty}$ denote the space of all bounded holomorphic functions on $D^{n}$ and $\partial_{j}=\partial / \partial z_{j}$.

Received 29th September, 1998

This research is partially supported by KOSEF(98-0701-03-01-5, 981-0102-014-2), KRF(98-1426) and GARC(98).

Copyright Clearance Centre, Inc. Serial-fee code: 0004-9729/99 \$A2.00+0.00. 
THEOREM 1. Let $f, g \in H^{\infty}$. Then $H_{\bar{f}}\left(A^{2}\right)$ and $H_{\bar{g}}\left(A^{2}\right)$ are mutually orthogonal if and only if either $\partial_{j} f$ or $\partial_{j} g$ is zero for each $j$ where $1 \leqslant j \leqslant n$.

Our next result is that the same is true for the Hardy space. To be more explicit, let $T$ be the boundary of $D$ and let $T^{n}$ denote the cartesian product of $n$ copies of $T$. Let $\sigma$ denote the usual normalised Haar measure on $T^{n}$ and $L^{p}\left(T^{n}\right)=L^{p}\left(T^{n}, \sigma\right)$. The Hardy space $H^{2}$ is the closed subspace of $L^{2}\left(T^{n}\right)$ consisting of all functions $u \in L^{2}\left(T^{n}\right)$ whose Poisson integrals are holomorphic on $D^{n}$. For $f \in L^{\infty}\left(T^{n}\right)$, the Hankel operator $h_{f}$ with symbol $f$ is defined by

$$
h_{f} u=u f-Q(u f)
$$

for $u \in H^{2}$. Here, $Q$ denotes the orthogonal projection from $L^{2}\left(T^{n}\right)$ onto $H^{2}$. Then, $h_{f}: H^{2} \rightarrow L^{2}\left(T^{n}\right)$ is a bounded linear operator and $h_{f}\left(H^{2}\right)$ is contained in $H^{2 \perp}$, the orthogonal complement of $H^{2}$ in $L^{2}\left(T^{n}\right)$. Here,

$$
h_{f}\left(H^{2}\right)=\left\{h_{f} u: u \in H^{2}\right\}
$$

denotes the image of $h_{f}$. On the hardy space $H^{2}$, we have the following.

THEOREM 2. Let $f, g \in H^{\infty}$. Then $h_{\bar{f}}\left(H^{2}\right)$ and $h_{\bar{g}}\left(H^{2}\right)$ are mutually orthogonal if and only if either $\partial_{j} f$ or $\partial_{j} g$ is zero for each $j$ where $1 \leqslant j \leqslant n$.

\section{Proofs}

Since every point evaluation is a bounded linear functional on $A^{2}$, there corresponds to every $a \in D^{n}$ a unique function $K_{a}$ in $A^{2}$ which has following reproducing property:

$$
f(a)=\left\langle f, K_{a}\right\rangle \quad \text { for all } f \in A^{2},
$$

where the notation $\langle$,$\rangle denotes the inner product in L^{2}\left(D^{n}\right)$ with respect to the measure $V$. The function $K_{a}$ is the well known Bergman kernel which has the explicit formula

$$
K_{a}(z)=\prod_{j=1}^{n} \frac{1}{\left(1-\bar{a}_{j} z_{j}\right)^{2}} \quad\left(z \in D^{n}\right)
$$

Here and elsewhere, we use the notation $z=\left(z_{1}, \cdots, z_{n}\right)$ for a point $z \in D^{n}$. We let $k_{a}$ denote the normalised kernel, namely,

$$
k_{a}(z)=\frac{K_{a}(z)}{\sqrt{K_{a}(a)}} \quad\left(a, z \in D^{n}\right) .
$$

For each $a=\left(a_{1}, \cdots, a_{n}\right) \in D^{n}$, we let $\varphi_{a}=\left(\varphi_{a_{1}}, \cdots, \varphi_{a_{n}}\right)$ where each $\varphi_{a_{i}}$ is the usual Möbius map on $D$ given by

$$
\varphi_{a_{i}}\left(z_{i}\right)=\frac{a_{i}-z_{i}}{1-\bar{a}_{i} z_{i}} \quad\left(z_{i} \in D\right)
$$


Then $\varphi_{a}$ is an automorphism of $D^{n}$ and $\varphi_{a} \circ \varphi_{a}$ is the identity on $D^{n}$. Since the real Jacobian of $\varphi_{a}$ is given by $\left|k_{a}\right|^{2}$, we have

$$
\int_{D^{n}} h \circ \varphi_{a} d V=\int_{D^{n}} h\left|k_{a}\right|^{2} d V
$$

whenever the integrals make sense. In particular, we have

$$
\int_{D^{n}} h\left|k_{a}\right|^{2} d V=h(a) \quad\left(a \in D^{n}\right)
$$

for functions $h$ integrable and holomorphic on $D^{n}$.

We say that a function $u$ is $n$-harmonic on $D^{n}$ if $u$ is harmonic in each variable separately. It turns out that $n$-harmonic functions can be characterised by a certain mean value property.

Lemma 3. Let $u \in L^{\infty}\left(D^{n}\right)$. Then $u$ is $n$-harmonic on $D^{n}$ if and only if

$$
\int_{D^{n}}\left(u \circ \varphi_{a}\right) d V=u(a)
$$

for every $a \in D^{n}$.

Proof: See [1, Theorem 3.1].

Lemma 4. For $f \in H^{\infty}$, we have

$$
H_{\bar{f}} k_{a}=(\bar{f}-\bar{f}(a)) k_{a}
$$

for all $a \in D^{n}$.

Proof: Let $f \in H^{\infty}$ and fix $z, a \in D^{n}$. By the reproducing property (1), we see

$$
P\left(\bar{f} k_{a}\right)(z)=\left\langle P\left(\bar{f} k_{a}\right), K_{z}\right\rangle=\left\langle\bar{f} k_{a}, K_{z}\right\rangle=\left\langle k_{a}, f K_{z}\right\rangle .
$$

On the other hand, we have

$$
\left\langle k_{a}, f K_{z}\right\rangle=\frac{\bar{f}(a) \overline{K_{z}(a)}}{\sqrt{K_{a}(a)}}=\bar{f}(a) k_{a}(z) .
$$

The proof is complete

We now prove Theorem 1.

Proof OF THEOREM 1: First, assume $H_{\bar{f}}\left(A^{2}\right)$ and $H_{\bar{g}}\left(A^{2}\right)$ are mutually orthogonal. It follows from (2) and Lemma 4 that

$$
\begin{aligned}
\left\langle H_{\bar{f}} k_{a}, H_{\bar{g}} k_{a}\right\rangle & =\left\langle H_{\bar{f}} k_{a}, \bar{g} k_{a}\right\rangle \\
& =\left\langle(\bar{f}-\bar{f}(a)) k_{a}, \bar{g} k_{a}\right\rangle \\
& =\int_{D^{n}} \bar{f} g\left|k_{a}\right|^{2} d V-\bar{f}(a) \int_{D^{n}} g\left|k_{a}\right|^{2} d V \\
& =\int_{D^{n}}(\bar{f} g) \circ \varphi_{a} d V-\bar{f}(a) g(a)
\end{aligned}
$$


for every $a \in D^{n}$. Since

$$
\left\langle H_{\bar{f}} k_{a}, H_{\bar{g}} k_{a}\right\rangle=0 \quad\left(a \in D^{n}\right)
$$

by assumption, it follows from Lemma 3 that $\bar{f} g$ is $n$-harmonic on $D^{n}$. Now, the desired result follows from the fact

$$
0=\partial_{j} \bar{\partial}_{j}(\bar{f} g)=\overline{\partial_{j} f} \partial_{j} g \quad(1 \leqslant j \leqslant n) .
$$

Conversely, assume either $\partial_{j} f$ or $\partial_{j} g$ is zero for each $j$. Then, by changing the coordinate system if needed, we may write the functions $f, g$ in the form

$$
f(z)=f\left(z_{1}, \cdots, z_{r}\right), \quad g(z)=g\left(z_{r+1}, \cdots, z_{n}\right)
$$

for some $1 \leqslant r \leqslant n$. Then, it is not hard to see that

$$
\left\langle g K_{a}, f K_{b}\right\rangle=\bar{f}\left(a_{1}, \cdots, a_{r}\right) g\left(b_{r+1}, \cdots, b_{n}\right) K_{a}(b)
$$

for every $a, b \in D^{n}$. This, together with (1) and Lemma 4, yields

$$
\begin{aligned}
\left\langle H_{\bar{f}} K_{a}, H_{\bar{g}} K_{b}\right\rangle & =\left\langle H_{\bar{f}} K_{a}, \bar{g} K_{b}\right\rangle \\
& =\left\langle\left(\bar{f}-\bar{f}\left(a_{1}, \cdots, a_{r}\right)\right) K_{a}, \bar{g} K_{b}\right\rangle \\
& =\left\langle g K_{a}, f K_{b}\right\rangle-\bar{f}\left(a_{1}, \cdots, a_{r}\right)\left\langle g K_{a}, K_{b}\right\rangle \\
& =\left\langle g K_{a}, f K_{b}\right\rangle-\bar{f}\left(a_{1}, \cdots, a_{r}\right) g\left(b_{r+1}, \cdots, b_{n}\right) K_{a}(b) \\
& =0
\end{aligned}
$$

for every $a, b \in D^{n}$. Now, since the set $\left\{K_{a}: a \in D^{n}\right\}$ spans a dense subset of $A^{2}$, we see that $H_{\bar{f}}\left(A^{2}\right)$ and $H_{\bar{g}}\left(A^{2}\right)$ are mutually orthogonal. The proof is complete.

We now turn to the case of Hardy space. As is well known, we can identify $u \in H^{2}$ with its holomorphic extension $u$ on $D^{n}$. With this identification, the reproducing kernel for $H^{2}$ is the Cauchy kernel $R_{z}$ whose explicit formula is given by

$$
R_{z}(\zeta)=\prod_{j=1}^{n} \frac{1}{1-\bar{z}_{j} \zeta_{j}} \quad\left(\zeta \in T^{n}, z \in D^{n}\right)
$$

and thus we can write the projection $Q$ as

$$
Q \varphi(z)=\left\langle\varphi, R_{z}\right\rangle
$$

for $\varphi \in L^{2}\left(T^{n}\right)$. Note that we still use the same notation $\langle$,$\rangle for the inner product on$ $L^{2}\left(T^{n}\right)$. See [2, Chapter 3] for details.

Let $r_{a}$ denote the normalised kernel defined by

$$
r_{a}=\prod_{j=1}^{n} \sqrt{1-\left|a_{i}\right|^{2}} R_{a}
$$


Note that $\left|r_{a}\right|^{2}$ is the Poisson kernel on $D^{n}$ and thus we have

$$
\int_{T^{n}} f\left|r_{a}\right|^{2} d \sigma=f(a) \quad\left(a \in D^{n}\right)
$$

for every $f \in H^{2}$. We also have the following whose proof is a trivial modification of that of Lemma 4 and thus omitted.

Lemma 5. For $f \in H^{\infty}$, we have

$$
h_{\bar{f}} r_{a}=(\bar{f}-\bar{f}(a)) r_{a}
$$

for all $a \in D^{n}$.

We now prove Theorem 2.

Proof of Theorem 2: First, assume $h_{\bar{f}}\left(H^{2}\right)$ and $h_{\bar{g}}\left(H^{2}\right)$ are mutually orthogonal. By (3) and Lemma 5 we have

$$
\begin{aligned}
\left\langle h_{\bar{f}} r_{a}, h_{\bar{g}} r_{a}\right\rangle & =\left\langle h_{\bar{f}} r_{a}, \bar{g} r_{a}\right\rangle \\
& =\left\langle(\bar{f}-\bar{f}(a)) r_{a}, \bar{g} r_{a}\right\rangle \\
& =\int_{T^{n}} \bar{f} g\left|r_{a}\right|^{2} d \sigma-\bar{f}(a) \int_{T^{n}} g\left|r_{a}\right|^{2} d \sigma \\
& =\int_{T^{n}} \bar{f} g\left|r_{a}\right|^{2} d \sigma-\bar{f}(a) g(a)
\end{aligned}
$$

for every $a \in D^{n}$. Since

$$
\left\langle H_{\bar{f}} r_{a}, H_{\bar{g}} r_{a}\right\rangle=0
$$

by the assumption, we have

$$
\bar{f}(a) g(a)=\int_{T^{n}} \bar{f} g\left|r_{a}\right|^{2} d \sigma
$$

for all $a \in D^{n}$. That is, the function $\bar{f} g$ is the Poisson integral of its boundary function $\bar{f} g \in L^{1}\left(T^{n}\right)$ and therefore is $n$-harmonic on $D^{n}$. See [2, Chapter 2]. Now, the result holds as in the proof of Theorem 1.

Conversely, assume either $\partial_{j} f$ or $\partial_{j} g$ is zero for each $j$. By exactly the same argument as in the proof of Theorem 1 , together with the fact the set $\left\{R_{a}: a \in D^{n}\right\}$ spans a dense subset of $H^{2}$, we see that $h_{\bar{f}}\left(H^{2}\right)$ and $h_{\bar{g}}\left(H^{2}\right)$ are mutually orthogonal. The proof is complete.

\section{REFERENCES}

[1] J. Lee, 'An invariant mean value property in the polydisc', (preprint).

[2] W. Rudin, Function theory in polydiscs (W.A. Benjamin, Inc., New York, Amsterdam, 1969). 
[3] J. Wang and Z. Wu, 'Images of Hankel operators', Integral Equations Operator Theory 28 (1997), 87-96.

Department of Mathematics

Korea University

Seoul 136-701

Korea

e-mail: choebr@semi.korea.ac.kr
Department of Mathematics

Mokpo National University

Chonnam 534-729

Korea

e-mail: yjlee@chungkye.mokpo.ac.kr 